\title{
Impact of exact exchange in the description of the electronic structure of organic charge-transfer molecular crystals
}

\author{
Alexandr Fonari, Christopher Sutton, Jean-Luc Brédas, ${ }^{*}$ and Veaceslav Coropceanu ${ }^{\dagger}$ \\ School of Chemistry and Biochemistry and Center for Organic Photonics and Electronics Georgia Institute of Technology Atlanta, \\ Georgia 30332-0400
}

(Received 14 July 2014; revised manuscript received 22 September 2014; published 21 October 2014)

\begin{abstract}
We evaluate the impact that the amount of nonlocal Hartree-Fock (\%HF) exchange included in a hybrid density functional has on the microscopic parameters (transfer integrals, band gaps, bandwidths, and effective masses) describing charge transport in high-mobility organic crystals. We consider both crystals based on a single molecule, such as pentacene, and crystals based on mixed-stack charge-transfer systems, such as dibenzo-TTF-TCNQ. In the pentacene crystal, the band gap decreases and the effective masses increase linearly with an increase in the amount of \%HF exchange. In contrast, in the charge-transfer crystals, while the band gap increases linearly, the effective masses vary only slightly with an increase in \%HF exchange. We show that the superexchange nature of the electronic couplings in charge-transfer systems is responsible for this peculiar evolution of the effective masses. We compare the density functional theory results with results obtained within the $G_{0} W_{0}$ approximation as a way of benchmarking the optimal amount of $\% \mathrm{HF}$ exchange needed in a given functional.
\end{abstract}

DOI: 10.1103/PhysRevB.90.165205

PACS number(s): 81.05.Fb, 71.20.Rv

\section{INTRODUCTION}

Electronic coupling is a key microscopic parameter in the description of the charge-transport properties of organic semiconductors. Large electronic couplings between $\pi$-conjugated molecules (i.e., large intermolecular wave function overlaps) are necessary for high charge-carrier mobilities [1]. There are two main theoretical approaches to derive the electronic couplings. The first is based on a molecular (localized) representation whereby the electronic coupling (or transfer integral $t$ ) between any two molecules can be evaluated from the quantum-mechanical solutions obtained on dimers (or complexes) consisting of these two molecules [1,2]. Alternatively, a momentum (delocalized) representation can be used, with the electronic couplings obtained from band structure calculations performed on the corresponding crystal using periodic boundary conditions. Similar to the role played by transfer integrals in the molecular picture, the widths of the valence [conduction] bands and the hole [electron] effective masses $\left(m_{\text {Eff }}\right)$ represent key charge-transport parameters for periodic systems. Although the actual charge-transport mechanism in organic molecular semiconductors remains a matter of debate, recent angle-resolved ultraviolet photoelectron spectroscopy measurements performed on rubrene and pentacene crystals point to a clear energy dispersion of the highest occupied electronic levels, indicating that the concept of electronic bands is valid for these systems [3-5]. We note that, when appropriately chosen, a simple tight-binding Hamiltonian can be used to relate the results obtained in the molecular picture to those in the momentum representation [1].

\footnotetext{
*jean-luc.bredas@kaust.edu.sa; Permanent address: Division of Physical Sciences and Engineering, King Abdullah University of Science and Technology, Thuwal 23955-6900, Kingdom of Saudi Arabia.

†coropceanu@gatech.edu
}

In the context of charge transport, the use of reliable quantum-mechanical approaches to evaluate the transfer integrals and the related electronic band structures are of critical importance. Over recent years, density functional theory (DFT) has been extensively used for the evaluation of the charge-transport microscopic parameters in organic semiconductors. Importantly, it was demonstrated that the electron-vibration couplings [6] and transfer integrals [7] are very much sensitive to the amount of nonlocal Hartree-Fock (\%HF) exchange included in the density functional. In an earlier paper, we have detailed the dependence of the transfer integral on \%HF exchange for pentacene and rubrene [7], which are two of the organic semiconducting crystals with the highest charge-carrier mobilities [8].

While interest in organic molecular semiconductors has largely focused on "one-component" systems based on a single molecule, recently "two-component" donoracceptor systems have also received significant attention both experimentally [8] and theoretically [9]. In particular, it was demonstrated that field-effect transistors based on the dibenzotetrathiafulvalene-7,7,8,8tetracyanoquinodimethane (DBTTF-TCNQ; see Fig. 1) crystal exhibit ambipolar behavior [10]. The DBTTF-TCNQ and other similar charge-transfer systems that display semiconducting properties usually crystallize in mixed-stack arrays in which the donor and acceptor molecules alternate along the stacking directions. Of particular interest to this paper, DBTTF-TCNQ has also been calculated previously to possess large transfer integrals for both holes and electrons [9].

In this paper, we compare how the electronic couplings, band gaps, bandwidths, and effective masses depend on the amount of \%HF exchange for pentacene and DBTTF-TCNQ, taken as representative examples of single-component and donor-acceptor crystals. We show that the unusual dependences found in DBTTF-TCNQ can be attributed to the superexchange nature of the electronic couplings in this class of materials. 

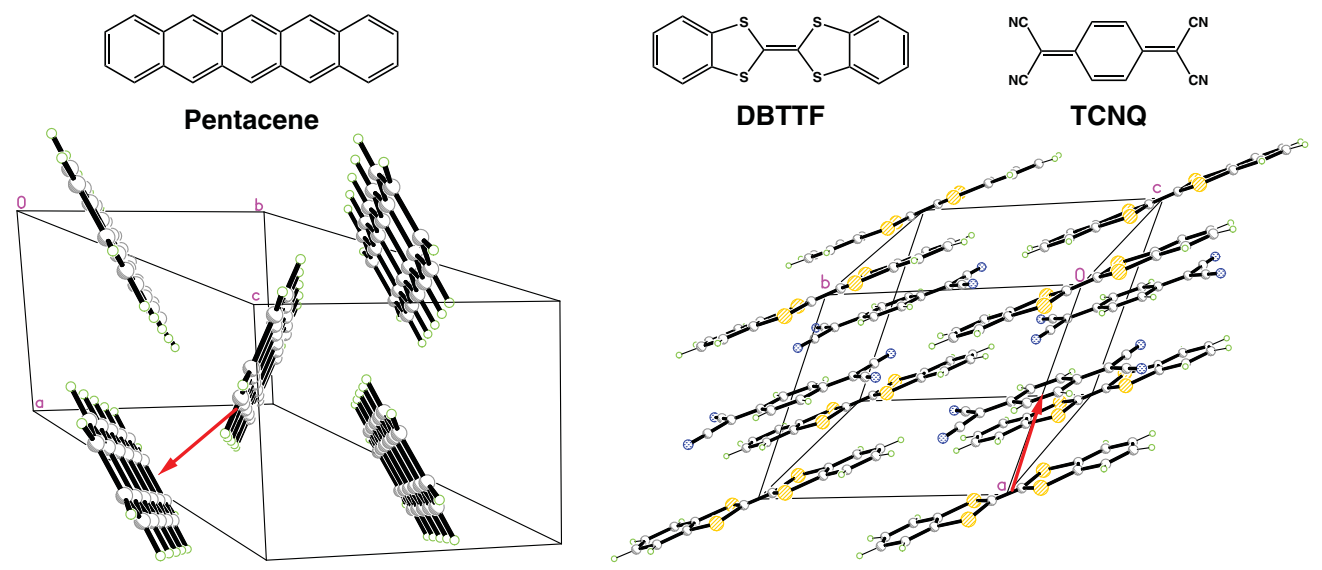

FIG. 1. (Color online) Chemical and crystal structures of the investigated systems: Pentacene [11] (left) and DBTTF-TCNQ [12] (right). Red arrows indicate the directions of the largest transfer integrals as well as the smallest effective masses for both holes and electrons.

\section{COMPUTATIONAL METHODOLOGY}

Electronic-structure calculations on both pentacene $(V$ polymorph with interlayer spacing of $14.1 \AA$ ) [11] and DBTTF-TCNQ [12] have been performed on the basis of their experimental crystal structures. The impact of the $\% \mathrm{HF}$ exchange has been investigated by using the $\alpha$ PerdewBurke-Ernzerhof ( $\alpha \mathrm{PBE})$ functional, based on the original PBE functional $[13,14]$, following the simple hybrid scheme proposed by Adamo and Barone [15]:

$$
E_{\alpha \mathrm{PBE}}=\alpha E_{X}^{\mathrm{HF}}+(1-\alpha) E_{X}^{\mathrm{PBE}}+E_{C}^{\mathrm{PBE}},
$$

where $\alpha=0.25$ corresponds to the PBE0 functional.

Direct electronic couplings were evaluated by using the fragment orbital approach [2] implemented in the developmental version of the NWChem package [16] in conjunction with the $6-31 G^{*}$ basis set. For the two-component system, effective hole [electron] electronic couplings were evaluated using donor-acceptor-donor [acceptor-donor-acceptor] triads, as we did in our previous work [9]. The CRYSTAL09 [17] was used to perform all calculations using the $\alpha \mathrm{PBE}$ functional combined with the $6-31 \mathrm{G}$ basis set. The Brillouin zone was sampled using a $\Gamma$-centered uniform $k$-point $6 \times 6 \times 6$ grid for the pentacene crystal and a $6 \times 6 \times 8$ grid for the DBTTFTCNQ crystal. The effective masses and their orientations were calculated by diagonalizing the inverse of the effective mass tensor $\left(m_{i j}^{-1}\right)$. The latter was obtained from the valence band maximum or conduction band minimum using a finitedifference method on a five-point stencil with a $0.01 \mathrm{Bohr}^{-1}$ step $(1$ Bohr $=0.529 \AA)$. Here, $G_{0} W_{0}$ calculations for the pentacene and DBTTF-TCNQ crystals were performed using the BerkeleyGW code [18], starting from the PBE-derived wave functions and energies obtained from Quantum Espresso [19] with norm-conserving Troullier-Martins pseudopotentials [20]. Core radii for the atoms were set to: 1.5 Bohr for $\mathrm{C}, 1.3$ Bohr for $\mathrm{H}, 1.4$ Bohr for $\mathrm{N}$, and 1.7 Bohr for $\mathrm{S}$. The kinetic energy cutoff for wave function was set to $60 \mathrm{Ry}(816.3 \mathrm{eV})$. The static dielectric matrix cutoff was set to $15 \mathrm{Ry}(204.1$ $\mathrm{eV}$ ). For the static dielectric matrix calculations (required for the evaluation of the screened Coulomb interaction $W$ within $G W$ ), the generalized plasmon model was used [21], with 464 unoccupied bands for both pentacene and DBTTF-TCNQ.
Here, $\Gamma$-centered uniform meshes of $4 \times 4 \times 2$ and $4 \times$ $4 \times 4 k$-points were employed for pentacene and DBTTFTCNQ, respectively. For the sake of comparison, in addition to the calculations that rely on an atomic orbital basis set (performed in CRYSTAL09), Vienna $A b$ initio Simulation Package (VASP) [22-25] calculations with plane-wave basis set (projector augmented wave [PAW] method) were also performed [26,27]. For both pentacene and DBTTF-TCNQ crystals, a plane wave energy cutoff of $800 \mathrm{eV}$ and a $2 \times 2 \times$ $2 k$-point mesh were employed.

\section{RESULTS AND DISCUSSION}

We first discuss the impact that the \%HF exchange in the $\alpha \mathrm{PBE}$ functional has on the band structure and effective masses of pentacene (see, Fig. 2 and Fig. 3). For the range between 0 to $50 \% \mathrm{HF}$ exchange, the valence [conduction] bandwidth increases by 0.207 [0.275] eV, i.e., by about $40 \%$. This result is consistent with the increase in electronic coupling evaluated from a dimer calculation, which we previously attributed to the fact that (semi)local functionals have an exchange-correlation potential that decays too quickly [7]. The effective masses for holes [electrons] were calculated at the valence band maximum $(0.375,0.5,0.075)$ [conduction band minimum $(0.39,0.5,0.5)]$ in reciprocal space; the smallest effective mass both for holes and electrons is estimated to be approximately along the herringbone direction (Fig. 1). The effective masses for holes [electrons] decrease almost linearly $\left(R^{2}=0.977[0.974]\right)$ from 2.011 to 1.406 [1.952 to 1.363 ] $m_{0}$ with increasing $\% \mathrm{HF}$ exchange, thus with an increasing transfer integral. This trend can be explained in the framework of the tight-binding model, where in the simple one-dimensional case, $m_{1 D}=\hbar^{2} / 2 t d^{2}$, where $d$ represents the intermolecular distance. The band gap also shows a linear increase $\left(R^{2}=0.999\right)$, by a factor of 3 from 0.83 to $3.08 \mathrm{eV}$ (Fig. 2), in agreement with previous findings [28,29]. These results underline that, depending on the $\% \mathrm{HF}$ exchange used in a given functional, the DFT estimates for the band gap, bandwidth, and effective mass can span a wide range.

More accurate results can be obtained by employing higher-level, first-principles electronic-structure methods. For 

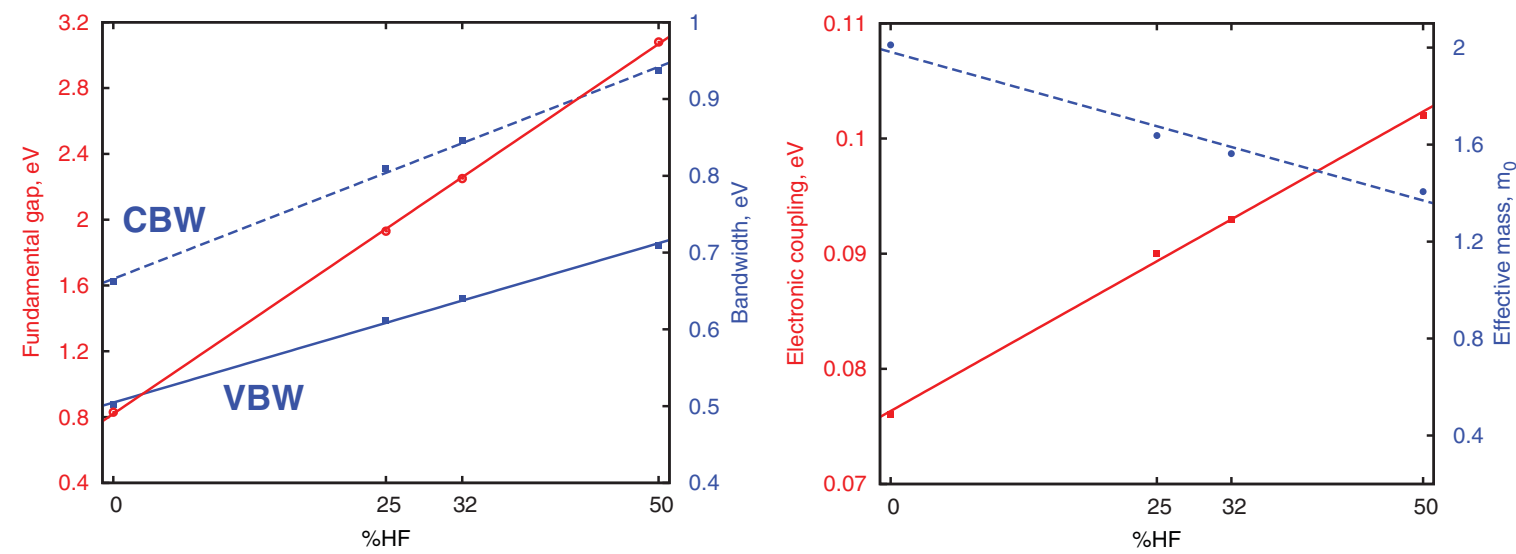

FIG. 2. (Color online) Dependence of the fundamental gap, valence and, conduction bandwidths on the \% HF exchange (left); dependence of the largest hole electronic coupling and smallest hole effective mass on $\% \mathrm{HF}$ exchange (right) in the crystalline pentacene.

instance, it has been shown that calculations performed within the $G W$ approximation [21,30,31] can reproduce the experimental fundamental gaps and valence and conduction bandwidths for both inorganic and organic semiconductors with a high accuracy, though at a much higher computational cost. Here, we have used the $G_{0} W_{0}$ results to calibrate the amount of $\mathrm{HF}$ exchange in the $\alpha \mathrm{PBE}$ functional for the present systems. We note that it has been shown in the case of isolated molecules and charge-transfer complexes that the $G_{0} W_{0}$ results depend to some extent on the DFT starting point [32,33]; here, for the sake of simplicity we consider only the results based on PBE-derived wave functions. The obtained $G_{0} W_{0}$ results are in very good agreement (see Table I) with those derived previously [34]. Tuning the \%HF exchange in $\alpha$ PBE to recover the fundamental gap obtained from $G_{0} W_{0}$ calculations (at the $E$ point in the Brillouin zone) results in a value of $32 \% \mathrm{HF}$ exchange. Importantly, the valence and conduction bandwidths derived by this tuned functional at the $E$ point and other $k$-points (Table I, Fig. 3 ) are also in excellent agreement with the $G_{0} W_{0}$ calculations.
We now turn to the two-component donor-acceptor systems that are expected to exhibit lower band gaps than the crystals of the individual components. In fact, when a pure functional or a functional with a low amount of the HF exchange $(<10 \%)$ is employed, the gap in DBTTF-TCNQ goes to zero (i.e., the system shows a metallic behavior) or demonstrates an unusual evolution that is no longer linear. A similar result was recently reported for another donor-acceptor system, i.e., tetrathiafulvalene- $p$-chloranil (TTF-CA) [35]. This behavior can be attributed to the well-known underestimation of the computed band gaps with (semi)local DFT functionals [36]. We also note that the calculations using a large \%HF exchange display convergence difficulties. Therefore, we only discuss below the results obtained within a range of $10-40 \% \mathrm{HF}$ exchange.

The valence [conduction] band structure as a function of $\%$ HF exchange is shown in Fig. 4. The effective masses for holes [electrons] were evaluated at the $(0.0,0.5,0.5)[(0.0,0.0$, $0.0)$ ] point in reciprocal space, with the smallest effective mass both for holes and electrons found along the stacking direction
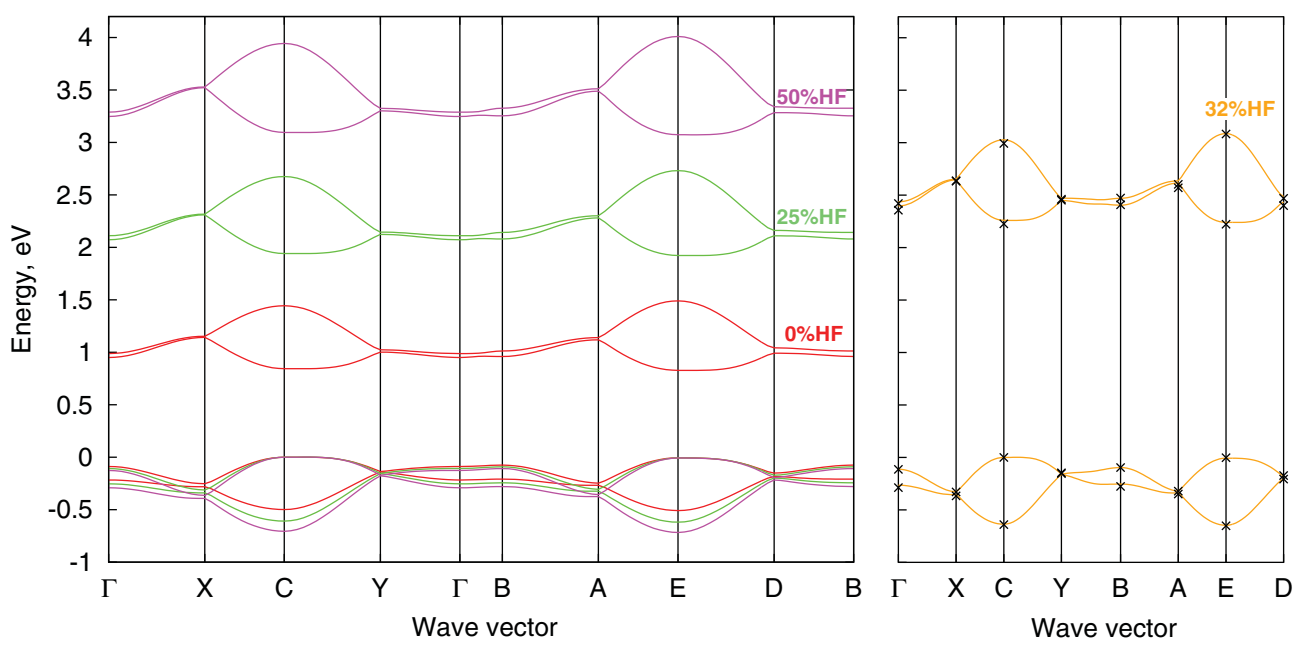

FIG. 3. (Color online) Left: Valence and conduction bands of pentacene obtained using the $\alpha \mathrm{PBE}$ functional with different $\% \mathrm{HF}$. Right: Comparison of $\alpha \mathrm{PBE}$ (solid lines) and $G_{0} W_{0}$ (crosses) results. The points of high symmetry in the first Brillouin zone are labeled as follows: $\Gamma=(0,0,0), X=(0.5,0,0), Y=(0,0.5,0), B=(0,0,0.5), C=(0.5,0.5,0), A=(0.5,0,0.5), E=(0.5,0.5,0.5)$, and $D=(0,0.5,0.5)$, all in crystallographic coordinates. The zero of energy corresponds to the top of the valence band, at the $C$ point. 
TABLE I. Fundamental gap $\left(E_{g}, \mathrm{eV}\right)$ at the $E$ point, smallest hole and electron effective masses ( $m_{\text {eff }}^{\text {Hole }}$ and $m_{\text {eff }}^{E l}$ in the units of mass of the electron at rest, $m_{0}$ ), valence (VBW) and conduction (CBW) bandwidths $(\mathrm{eV})$ at the $E$ point.

\begin{tabular}{lccccccc}
\hline \hline$\%$ HF & $E_{g}$ & $m_{\text {eff }}^{\text {Hole }}$ & $m_{\text {eff }}^{E 1}$ & VBW & CBW & $t^{\text {Hole }}$ & $t^{E 1}$ \\
\hline 0 & 0.83 & 2.011 & 1.952 & 0.502 & 0.662 & 0.076 & 0.080 \\
25 & 1.93 & 1.637 & 1.581 & 0.612 & 0.809 & 0.090 & 0.094 \\
32 & 2.25 & 1.563 & 1.511 & 0.640 & 0.846 & 0.093 & 0.098 \\
50 & 3.08 & 1.406 & 1.363 & 0.709 & 0.937 & 0.102 & 0.107 \\
$G_{0} W_{0}$ & 2.22 & & & 0.648 & 0.859 & & \\
$G_{0} W_{0}[34]$ & 1.9 & & & 0.54 & 0.67 & & \\
\hline \hline
\end{tabular}

( $a$ axis; Fig. 1). The striking difference compared to the onecomponent systems is the very weak evolution of the valence [conduction] bandwidths: 0.029 [0.033] eV within the range of $10-40 \% \mathrm{HF}$ exchange. As a consequence, there occurs only a very slight increase in the effective masses (as opposed to the significant decrease in effective mass with $\% \mathrm{HF}$ observed in pentacene) for both holes [0.299 to $0.407 \mathrm{~m}_{0}$ ] and electrons [0.315 to $0.461 m_{0}$, see Table II. We note that very similar values for bandwidths are found as well when using the PAW basis set, which confirms that the evolution is not related to the choice of basis set. In contrast to the very slight evolution of the bandwidths, an increase in \% HF leads, as in the case of pentacene, to a linear increase of the fundamental gap (by a factor of $\sim 3$, from 0.50 to $1.67 \mathrm{eV}$, over the $10-40 \% \mathrm{HF}$ range; Fig. 4 ).

The significant difference in the manifestation of the $\% \mathrm{HF}$ exchange on the band structures of pentacene and DBTTFTCNQ can be attributed to the difference in the nature of electronic couplings in one- and two-component systems. In pentacene, the electronic couplings depend on the direct, through-space wave function overlap; in contrast, in DBTTFTCNQ, the electronic couplings have a superexchange nature,
TABLE II. Fundamental gap $\left(E_{g}, \mathrm{eV}\right)$ at the $\Gamma$ point; the smallest hole and electron effective mass ( $m_{\mathrm{eff}}^{\text {Hole }}$ and $m_{\mathrm{eff}}^{E 1}$ in units of the mass of the electron at rest, $\left.m_{0}\right)$; valence bandwidth $(\mathrm{VBW}, \mathrm{eV})$ at the $A$ point and conduction bandwidth $(\mathrm{CBW}, \mathrm{eV})$ at the $E$ point for the DBTTF-TCNQ crystal.

\begin{tabular}{|c|c|c|c|c|c|c|c|c|}
\hline \multirow{2}{*}{$\begin{array}{l}\% \mathrm{HF} \\
\text { Basis set }\end{array}$} & \multicolumn{2}{|c|}{$E_{g}$} & \multirow{2}{*}{$\begin{array}{c}m_{\mathrm{eff}}^{\mathrm{Hole}} \\
\mathrm{AO}\end{array}$} & \multirow{2}{*}{$\begin{array}{l}m_{\mathrm{eff}}^{E \mathrm{l}} \\
\mathrm{AO}\end{array}$} & \multicolumn{2}{|c|}{ VBW } & \multicolumn{2}{|c|}{ CBW } \\
\hline & $\mathrm{AO}$ & PAW & & & $\mathrm{AO}$ & PAW & $\mathrm{AO}$ & PAW \\
\hline 10 & 0.50 & 0.44 & 0.299 & 0.315 & 0.436 & 0.473 & 0.416 & 0.451 \\
\hline 25 & 0.98 & 1.06 & 0.338 & 0.368 & 0.465 & 0.474 & 0.435 & 0.441 \\
\hline $33^{\mathrm{a}} / 31^{\mathrm{b}}$ & 1.33 & 1.34 & 0.372 & 0.413 & 0.457 & 0.469 & 0.421 & 0.430 \\
\hline 40 & 1.67 & 1.75 & 0.407 & 0.461 & 0.442 & 0.456 & 0.402 & 0.410 \\
\hline$G_{0} W_{0}$ & \multicolumn{2}{|c|}{1.32} & & & \multicolumn{2}{|c|}{0.364} & \multicolumn{2}{|c|}{0.344} \\
\hline
\end{tabular}

${ }^{\mathrm{a}} \mathrm{AO}$ (atomic orbitals).

${ }^{b}$ PAW (projector augmented wave).

i.e., the electronic coupling for holes [electrons] results from the mixing of the frontier orbitals of two closest donor [acceptor] molecules with the frontier orbitals of the "bridging" acceptor [donor] molecule [9]. In the context of such a superexchange mechanism, both the electronic coupling and the energy difference between the relevant frontier molecular orbitals on adjacent donor and acceptor molecules have to be taken into account.

Using perturbation theory and assuming that only the pathway described above contributes to the superexchange mechanism, the effective transfer integrals for holes and electrons along the stacking directions are equal and are given by [9]:

$$
t_{H_{D}-H_{D}}^{\mathrm{eff}}=t_{L_{A}-L_{A}}^{\mathrm{eff}}=\frac{t_{H_{D}-L_{A}}^{2}}{\Delta E} .
$$

Here, $t_{H_{D}-L_{A}}$ is the electronic coupling between the highest occupied molecular orbital (HOMO) of a donor and the lowest
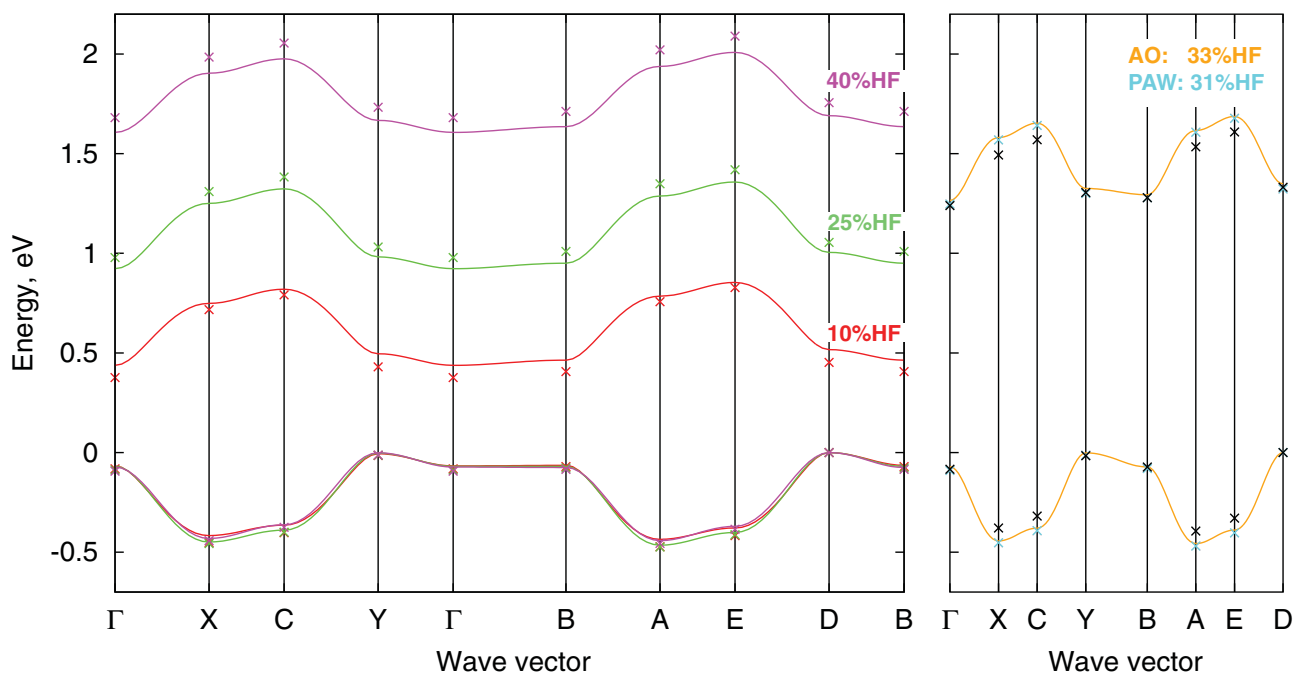

FIG. 4. (Color online) Left: Valence and conduction bands of DBTTF-TCNQ obtained using the $\alpha$ PBE functional with different amount of $\% \mathrm{HF}$ and different basis sets: atomic orbitals (solid lines) and PAWs (colored crosses). Right: Comparison of $\alpha \mathrm{PBE}$ and $G_{0} W_{0}$ (black crosses). The points of high symmetry in the first Brillouin zone are labeled as follows: $\Gamma=(0,0,0), X=(0.5,0,0), Y=(0,0.5,0), B=(0,0,0.5)$, $C=(0.5,0.5,0), A=(0.5,0,0.5), E=(0.5,0.5,0.5)$, and $D=(0,0.5,0.5)$, all in crystallographic coordinates. The zero of energy corresponds to the top of the valence band, at the $D$ point. 

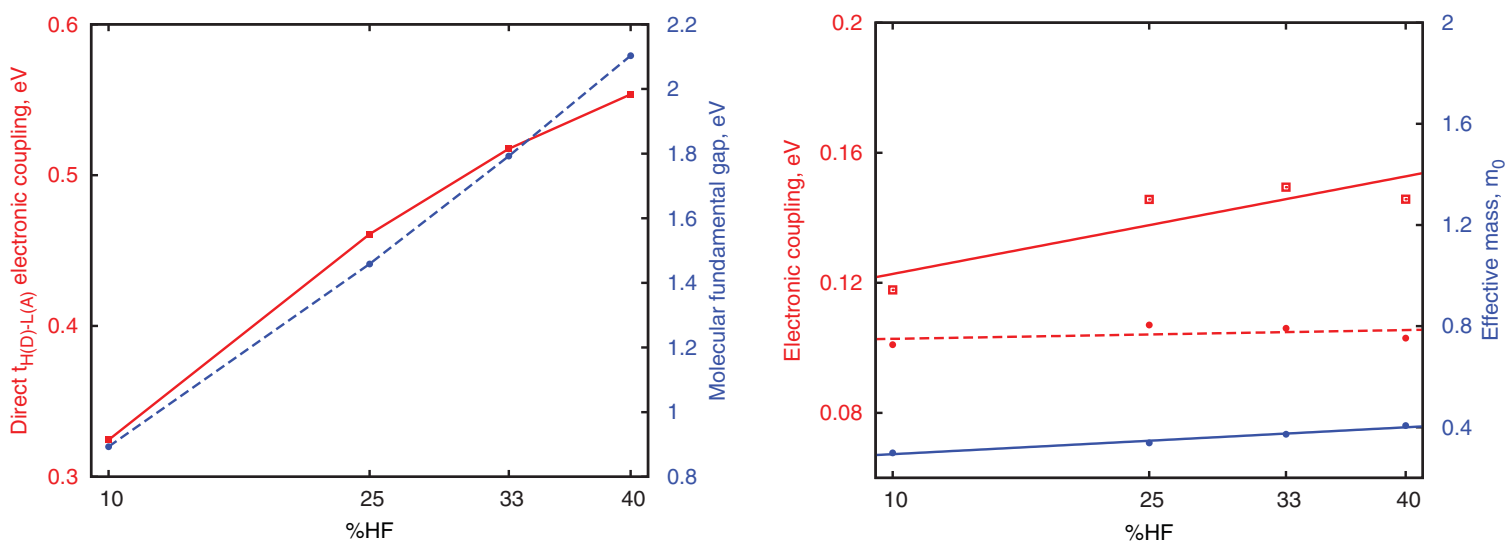

FIG. 5. (Color online) (a) Dependence of the donor-acceptor electronic coupling and molecular fundamental gap on the \%HF exchange; (b) dependence of the largest effective hole electronic coupling based on the energy splitting approach (dashed line) and using Eq. (2) (solid red line) and the smallest hole effective mass (solid blue line), on the \%HF exchange in the DBTTF-TCNQ crystal.

occupied molecular orbital (LUMO) of the adjacent acceptor; $\Delta E$ represents the energy of the charge-transfer state of the donor-acceptor dyad, which can be approximated by the energy of the fundamental gap. Thus, since both the band gap and the donor-acceptor electronic coupling concomitantly increase with the increase in \% $\mathrm{HF}$ exchange, the effective transfer integrals $\left(t_{H_{D}-H_{D}}\right.$ and $\left.t_{L_{A}-L_{A}}\right)$ are less sensitive to the choice of $\% \mathrm{HF}$ (see Fig. 5) than $t_{H_{D}-L_{A}}$ and $\Delta E$ individually. We note that Eq. (2) applies only in the perturbation limit, that is, when $t \ll \Delta E$. In the case where $t_{H_{D}-L_{A}}$ and $\Delta E$ are comparable, which is the situation in DBTTF-TCNQ [see Fig. 5(a)], the effective transfer integrals are expected to exhibit a more complex dependence on $t_{H_{D}-L_{A}}$ and $\Delta E$. Indeed, the values of $t_{H_{D}-H_{D}}$ derived by means of the nonperturbative energy splitting approach [9] show a much weaker dependence on \% HF exchange than those derived via Eq. (1) [see Fig. 5(b)].

Using $G_{0} W_{0}$, the fundamental gap for DBTTF-TCNQ is calculated to be $1.32 \mathrm{eV}$. When tuning the amount of $\% \mathrm{HF}$ exchange in $\alpha \mathrm{PBE}$ to recover the $G_{0} W_{0}$ results, a band gap of $1.33 \mathrm{eV}$ is obtained with $33 \% \mathrm{HF}$ exchange using an atomic orbital basis set and of $1.34 \mathrm{eV}$ with $33 \% \mathrm{HF}$ exchange using PAW. We note, however, that in contrast to the pentacene where the tuned $\alpha \mathrm{PBE}$ calculations can match the $G_{0} W_{0}$ results in the whole Brillouin zone, in the case of DBTTFTCNQ some discrepancy is observed between the bandwidths computed along the charge-transfer and other directions. This discrepancy can be traced back to the deficiency of the standard semilocal and global hybrid exchange-correlation functionals that do not provide the correct evaluation of the long-range Coulomb interaction that result in substantial errors for long-range charge-transfer excitations [37]. It has been suggested recently that an improved description of chargetransfer states can be obtained by employing range-separated hybrid functionals, especially those with system-tuned rangeseparation parameters [38]. While there are now multiple examples of applying these functionals to the donor-acceptor complexes $[39,40]$, their application to donor-acceptor crystals is still missing. Such investigations in our group are now underway, and the related results will be reported elsewhere.

\section{CONCLUSIONS}

We have evaluated the dependence on the amount of HF exchange included in the density functional of the chargetransport parameters in two organic semiconductor crystals, pentacene and DBTTF-TCNQ. We have found that in onecomponent systems such as pentacene, the fundamental gap, bandwidths, and effective masses vary significantly and in a linear fashion as a function of $\% \mathrm{HF}$ exchange. We have confirmed the superexchange mechanism of the electronic coupling in mixed-stack, donor-acceptor systems such as DBTTF-TCNQ. The superexchange nature of the electronic coupling affects the dependence of the electronic properties on \% HF exchange; in particular, with an increase in the $\% \mathrm{HF}$ exchange included in the functional, the bandwidths and effective masses remain nearly constant, in marked contrast to the evolution observed in one-component systems. We have also found that in both types of systems, the band gaps show a similar evolution, increasing by a factor of at least 3 when increasing the percentage of HF exchange from $\sim 0$ to $\sim 50 \%$. This is particularly important to realize when considering two-component systems, such as DBTTF-TCNQ which is calculated to be metallic when small (or no) amount of HF exchange is included in the functional. We have also shown that, by changing the amount of the HF exchange in the $\alpha \mathrm{PBE}$ functional, the $\alpha \mathrm{PBE}$ band gap can be tuned to match the $G_{0} W_{0}$ band gap, with the resulting bandwidths also in good agreement with those obtained from $G_{0} W_{0}$. Interestingly, the optimum $\% \mathrm{HF}$ exchange found here (in the range $31-$ $33 \%$ ) is very similar to the recently developed PBE0-1/3 functional, which is parameterized with $33 \% \mathrm{HF}$ exchange and outperforms the original PBE0 functional with 25\%HF exchange for a variety of molecular electronic properties [41].

\section{ACKNOWLEDGMENTS}

This work has been funded by the National Science Foundation (NSF) under Award No. DMR-1105147 and by the US Army Research Laboratory and US Army Research Office under Contract/Grant No. W911NF-13-1-0387. 
The computational resources have been made partly available via the Chemistry Research Instrumentation and Facilities (CRIF) Program of the NSF under Award
No. CHE-0946869. We are grateful to Dr. Gjergji Sini and Dr. Sahar Sharifzadeh for stimulating discussions and technical assistance.
[1] V. Coropceanu, J. Cornil, D. A. da Silva Filho, Y. Olivier, R. Silbey, and J. L. Bredas, Chemical Reviews 107, 926 (2007).

[2] E. F. Valeev, V. Coropceanu, D. A. da Silva Filho, S. Salman, and J. L. Bredas, J. Am. Chem. Soc. 128, 9882 (2006).

[3] S.-I. Machida, Y. Nakayama, S. Duhm, Q. Xin, A. Funakoshi, N. Ogawa, S. Kera, N. Ueno, and H. Ishii, Phys. Rev. Lett. 104, 156401 (2010).

[4] R. C. Hatch, D. L. Huber, and H. Höchst, Phys. Rev. Lett. 104, 047601 (2010).

[5] N. Koch, A. Vollmer, I. Salzmann, B. Nickel, H. Weiss, and J. P. Rabe, Phys. Rev. Lett. 96, 156803 (2006).

[6] R. S. Sánchez-Carrera, V. Coropceanu, D. A. da Silva Filho, R. Friedlein, W. Osikowicz, R. Murdey, C. Suess, W. R. Salaneck, and J.-L. Brédas, J. Phys. Chem. B 110, 18904 (2006).

[7] C. Sutton, J. S. Sears, V. Coropceanu, and J. L. Bredas, J. Phys. Chem. Lett. 4, 919 (2013).

[8] T. Hasegawa and J. Takeya, Sci. Tech. Adv. Mater. 10, 024314 (2009).

[9] L. Zhu, Y. Yi, Y. Li, E.-G. Kim, V. Coropceanu, and J. L. Bredas, J. Am. Chem. Soc. 134, 2340 (2012).

[10] Y. Takahashi, T. Hasegawa, Y. Abe, Y. Tokura, and G. Saito, Appl. Phys. Lett. 88, 073504 (2006).

[11] T. Siegrist, C. Besnard, S. Haas, M. Schiltz, P. Pattison, D. Chernyshov, B. Batlogg, and C. Kloc, Adv. Mater. 19, 2079 (2007).

[12] T. J. Emge, F. M. Wiygul, J. S. Chappell, A. N. Bloch, J. P. Ferraris, D. O. Cowan, and T. J. Kistenmacher, Mol. Cryst. Liq. Cryst. 87, 137 (1982).

[13] J. P. Perdew, K. Burke, and M. Ernzerhof, Phys. Rev. Lett. 77, 3865 (1996).

[14] J. P. Perdew, K. Burke, and M. Ernzerhof, Phys. Rev. Lett. 78, 1396 (1997).

[15] C. Adamo and V. Barone, J. Chem. Phys. 110, 6158 (1999).

[16] M. Valiev, E. J. Bylaska, N. Govind, K. Kowalski, T. P. Straatsma, H. J. J. Van Dam, D. Wang, J. Nieplocha, E. Apra, T. L. Windus, and W. A. de Jong, Comput. Phys. Commun. 181, 1477 (2010).

[17] R. Dovesi, R. Orlando, B. Civalleri, C. Roetti, V. R. Saunders, and C. M. Zicovich-Wilson, Z. Kristallogr. - Cryst. Mater. 220, 571 (2005).
[18] J. Deslippe, G. Samsonidze, D. A. Strubbe, M. Jain, M. L. Cohen, and S. G. Louie, Comput. Phys. Commun. 183, 1269 (2012).

[19] P. Giannozzi, S. Baroni, N. Bonini, M. Calandra, R. Car, C. Cavazzoni, D. Ceresoli, G. L. Chiarotti, M. Cococcioni, I. Dabo, A. D. Corso, S. d. Gironcoli, S. Fabris, G. Fratesi, R. Gebauer, U. Gerstmann, C. Gougoussis, A. Kokalj, M. Lazzeri, L. Martin-Samos et al., J. Phys.: Condens. Matter 21, 395502 (2009).

[20] N. Troullier and J. L. Martins, Phys. Rev. B 43, 1993 (1991).

[21] M. S. Hybertsen and S. G. Louie, Phys. Rev. B 34, 5390 (1986).

[22] G. Kresse and J. Furthmüller, Comput. Mat. Sci. 6, 15 (1996).

[23] G. Kresse and J. Furthmüller, Phys. Rev. B 54, 11169 (1996).

[24] G. Kresse and J. Hafner, Phys. Rev. B 47, 558 (1993).

[25] G. Kresse and J. Hafner, Phys. Rev. B 49, 14251 (1994).

[26] P. E. Blöchl, Phys. Rev. B 50, 17953 (1994).

[27] G. Kresse and D. Joubert, Phys. Rev. B 59, 1758 (1999).

[28] S. Refaely-Abramson, S. Sharifzadeh, M. Jain, R. Baer, J. B. Neaton, and L. Kronik, Phys. Rev. B 88, 081204 (2013).

[29] W. F. Perger, Chem. Phys. Lett. 368, 319 (2003).

[30] L. Hedin, Phys. Rev. 139, A796 (1965).

[31] S. Sharifzadeh, A. Biller, L. Kronik, and J. B. Neaton, Phys. Rev. B 85, 125307 (2012).

[32] T. Korzdorfer and N. Marom, Phys. Rev. B 86, 041110 (2012).

[33] V. Atalla, M. Yoon, F. Caruso, P. Rinke, and M. Scheffler, Phys. Rev. B 88, 165122 (2013).

[34] M. L. Tiago, J. E. Northrup, and S. G. Louie, Phys. Rev. B 67, 115212 (2003).

[35] G. Giovannetti, S. Kumar, A. Stroppa, J. van den Brink, and S. Picozzi, Phys. Rev. Lett. 103, 266401 (2009).

[36] K. Burke, J. Chem. Phys. 136, 150901 (2012).

[37] A. J. Cohen, P. Mori-Sánchez, and W. Yang, Chem. Rev. 112, 289 (2011).

[38] T. Stein, L. Kronik, and R. Baer, J. Am. Chem. Soc. 131, 2818 (2009).

[39] G. Sini, J. S. Sears, and J.-L. Brédas, J. Chem. Theory Comput. 7, 602 (2011).

[40] C. R. Zhang, J. S. Sears, B. Yang, S. G. Aziz, V. Coropceanu, and J. L. Bredas, J. Chem. Theory Comput. 10, 2379 (2014).

[41] C. A. Guido, E. Brémond, C. Adamo, and P. Cortona, J. Chem. Phys. 138, 021104 (2013). 